\title{
Heterochrony in helicoid spiral cones: a computer model for demonstrating heterochronic evolution
}

\author{
Andrew Robert Henry Swan
}

\begin{abstract}
A modification of Raup's classic helicoid logarithmic spiral cone model allows the incorporation of parameters for ontogenetic change in the main geometrical parameter values. The resulting allometric structures can be modified by simulated heterochronic increments so that the effects of neoteny, acceleration, progenesis and hypermorphosis can be produced. Experiments with the model, named HETEROSIM, produce suites of forms that are consistent with those that occur within Families of the Class Gastropoda, and hence heterochrony is a plausible hypothesis for evolution in those taxa. In addition to applications in evolutionary research, the model is a valuable pedagogic tool in teaching advanced palaeontology and evolution.
\end{abstract}

Andrew Robert Henry Swan. School of Geography, Geology and the Environment, Faculty of Science, Engineering and Computing, Kingston University, Penrhyn Road, Kingston upon Thames, Surrey KT1 2EE, United Kingdom Email: swan@kingston.ac.uk

Keywords: Heterochrony; computer model; morphospace; gastropods

\section{INTRODUCTION}

In heterochronic evolution, ancestor and descendent differ in the rate and/or timing of ontogenetic change (Gould, 1977; Alberch et al., 1979). This mode of evolution has been postulated as important in all groups of organisms, with case studies based on palaeontological evidence involving trilobites, gastropods, ammonites, plants, rodents and primates (see, for example, McKinney, 1988). This paper focusses on molluscan shell shapes, which have often been the subject of heterochronic interpretations: for example work by Gould (1969), Tissot (1988), Allman (1994) and Seuss et al. (2012) makes it clear that heteroch- rony is significant in gastropod evolution; and papers by Swan (1988), Hammer and Bucher (2005) and Gerber (2011) demonstrate heterochrony in shelled cephalopods. However, modelling of molluscan shells has been dominated by the helicoid logarithmic spiral cone model of David Raup (1966). Raup's simulations, as a consequence of the logarithmic model, are isometric: there is no change in the shape parameters during ontogeny. Consequently, heterochrony applied to such forms cannot result in evolutionary change in shape. This paper demonstrates the effect of heterochronic modes on an allometric model in which the parameters of a helicoid spiral cone vary through ontogeny. Experiments with the computer

PE Article Number: 18.1.2T

Copyright: Palaeontological Association April 2015

Submission: 5 September 2014. Acceptance: 11 March 2015

Swan, Andrew Robert Henry. 2015. Heterochrony in helicoid spiral cones: a computer model for demonstrating heterochronic evolution. Palaeontologia Electronica 18.1.2T: 1-11.

palaeo-electronica.org/content/2015/1120-gastropod-heterochrony-model 
model, named HETEROSIM, show that a range of forms, especially of terrestrial gastropods, are consistent with a heterochronic origin, and the model can be used as a pedagogic tool to demonstrate heterochronic modes.

\section{HETEROCHRONIC MODES}

Alberch et al. (1979) rationalised the terminology of heterochronic modes as follows:

Paedomorphosis. The adult form of the descendant resembles the juvenile form of the ancestor. This can result from neoteny, where the rate of ontogenetic morphological change is slower in the descendant, or by progenesis, where ontogenetic change terminates early (often accompanied by early attainment of sexual maturity and short life spans).

Peramorphosis. The juvenile form of the descendant resembles the adult form of the ancestor, and the adult form of the descendant results from extrapolation of ontogenetic trends. This can result from acceleration, where the rate of ontogenetic morphological change is faster in the descendant, or by hypermorphosis, where ontogenetic change continues for a longer time.

Proportioned dwarfism and gigantism. Here the ontogenetic shape change is unaffected by evolution; ancestor and descendant differ in terms of size only. These modes can be modelled by scale change alone; this is mundane and is not further considered here. However, note that size change accompanying shape change is a common consequence of paedomorphic and peramorphic evolution.

\section{MODELLING MOLLUSCAN SHELLS}

The discovery that shells such as those of Nautilus can be modelled by logarithmic spirals dates back at least to the eighteenth century. Although measurement and analysis were used subsequently to demonstrate logarithmic spirals in a variety of molluscs, it was Raup's (1966) work that allowed the wider recognition of many molluscan (and brachiopod) shells as helicoid logarithmic spiral cones. Raup isolated four parameters that defined such structures (refer to Figure 1):

$W$ : Whorl expansion rate (ratio of whorl diameters separated by one rotation about the coiling axis):

$$
W=b^{\prime} / \mathrm{b}
$$

$D$ : Diameter of umbilicus (as a proportion of total diameter):

$$
D=\frac{a}{a+b}
$$

S: Whorl shape (elliptical ratio: diameter parallel to coiling axis divided by diameter perpendicular to coiling axis).

T: Whorl translation (distance centre of whorl translates parallel to coiling axis in one rotation, divided by increase in distance of centre of whorl from coiling axis):

$$
T=c / x
$$

In the current study, a modification of the "whorl translation" parameter is used; this will be symbolised by $T$ :

$$
T^{\prime}=\frac{c}{\left(a+\frac{b}{2}\right)}
$$

(distance centre of whorl translates parallel to coiling axis in one rotation, divided by distance of whorl centre from coiling axis).

Raup's $T$ has the attractive property that all shapes with the same $T$ have the same apical angle, regardless of the value of $W$. However, this is achieved by connecting the two parameters, as $x$ is dependent on $W$. In heterochronic studies, it is useful to treat separate attributes as independent, or dissociated, from each other, at least in principle. So, we can regard the two attributes here as "expansion" and "translation", rather than, effectively, "expansion" and "apical angle" in Raup's formulation. As we will see in the experiments documented below, the use of $T$ rather than $T$ allows the modelling of forms that occur in nature

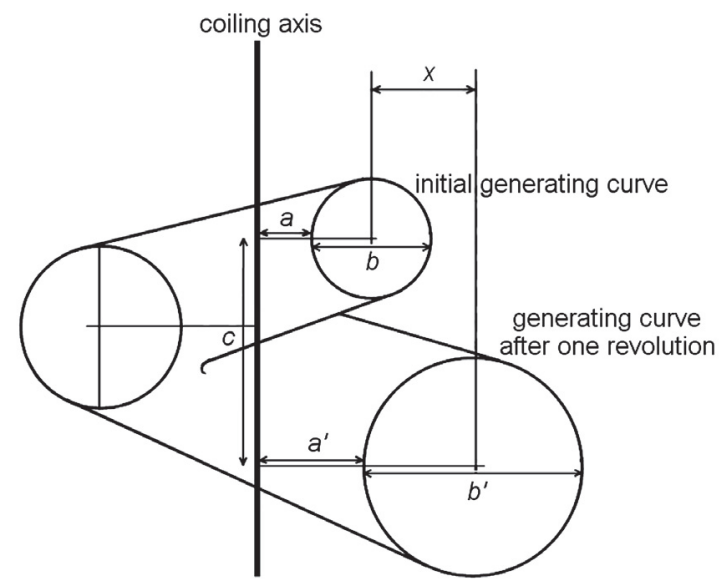

FIGURE 1. Raup's definition of the shell dimensions on which the equations for parameters $W, D$ and $T$ are derived. Based on Raup (1966). 
in which whorl expansion ceases through ontogeny, while translation continues.

The four "Raupian" parameters define a parameter space ("morphospace") in which can be recognised separate (or partially overlapping) regions of occupancy by members of molluscan classes Cephalopoda, Gastropoda and Bivalvia, and members of phylum Brachiopoda. This model has been found to be applicable, at least approximately, to many extant and fossil molluscan and brachiopod shells (Swan, 1999, 2001), and has been the basis of models that add further parameters, for example aperture orientation (Noshita et al., 2012). "Raupian" shapes are isometric, and hence shells fitting this model do not change shape with growth. Although this might seem to be a reasonable pattern of growth, reflecting perhaps an unchanging mode of life through ontogeny, it is in fact surprising, as animals typically do change their mode of life in ontogeny (e.g., they may occupy different niches and exploit different food sources), and even a consistent mode of life might demand allometric changes in response to physical effects of inevitable area:volume ratio changes.

A property of "Raupian" shapes that assists in their recognition is that, in a profile view (perpendicular to the coiling axis), a straight line can be found that is tangential to all whorls (Figure 2.1-2).

Allometric growth in molluscs has been recognised by analysis of ammonites (e.g., Kant and Kullman, 1978) and is easy to observe in many gastropods, especially terrestrial forms (Figure 2.34). Allometric computer models have consequently been developed by several authors. Okamoto (1988a) used Frenet moving frame analysis, which allows an elliptical frame to move and expand freely in three dimensional (3D) space, with parameters for expansion, torsion and curvature that have values that can change continuously. Any arbitrarily curved tube or cone can be modelled. Okamoto (1988b) used this approach to great advantage in hydrostatic modelling of heteromorph ammonites, as the method facilitates calculation of centres of buoyancy and gravity. A difficulty of the method is the intricacy involved in finding the complete ontogenetic series of parameters that best represents an actually occurring form. Rice (1998) and Hammer and Bucher (2005) introduced models where movement of the generating curve is determined by vectors on its perimeter; these are also models with high-dimensional parameter space. Rice (1998) demonstrated that change in rate of shell production (which could be heterochronic) could explain substantial evolutionary change in gastropod morphology. Hammer and Bucher (2005) were able to demonstrate allometric changes in ammonite apertures that could be subject to heterochronic change.

The allometric model used here is relatively simple (with low parameter dimensionality) and is based on Raup's model, but with four parameters $\left(W, D, S\right.$ and $\left.T^{\prime}\right)$ permitted to change linearly. For the purposes of the present paper, only $W$ and $T$, are subject to ontogenetic change. The additional parameters are $\Delta W$ and $\Delta T^{\prime} . W$ and $T^{\prime}$ ' therefore function merely as the initial values; they will be distinguished as $W_{0}$ and $T^{\prime}{ }_{0}$. If the radius of the $i^{\text {th }}$ whorl is $b_{i}$, then in Raup's model:

$$
b_{i}=W b_{i-1}
$$

whilst in the current model:

$$
b_{i}=\left(W_{0}+i \Delta W\right) b_{i-1}
$$

Similarly, in an isometric model the amount of translation in one rotation $c_{i}$ is given by:

$$
c_{i}=T^{\prime}\left(a_{i-1}+\frac{b_{i-1}}{2}\right)
$$

whilst in the current model:

$$
c_{i}=\left(T^{\prime}{ }_{o}+i \Delta T^{\prime}\right) \cdot\left(a_{i-1}+\frac{b_{i-1}}{2}\right)
$$

There is no intention here to justify this model as a valid model of growth of molluscan shells. It remains uncertain how the genetics that control molluscan growth correlate with the form of the final adult shell, or how variation in shell geometry relates to genetic variation (this has been discussed by Rice [1998] and Urdy and Chirat [2006]). The organism will probably not have suites of alleles that correlate with such geometrical parameters. Indeed, although the coiling axis of gastropod shells seems to be a fundamental feature (it is readily locatable and is straight with remarkable consistency), molluscs presumably aren't "aware" of, and don't refer to, the coiling axis when growing and secreting shells. Rather, the model is intended here to quantify in an approximate way ontogenetic change similar to that observed in real organisms, and then to experiment with simulated heterochrony, making predictions of morphologies that may result.

\section{MODELLING HETEROCHRONIC EVOLUTION}

Using the above model, "descendants" can be derived from "ancestors" through simulated heterochronic modes as follows: 

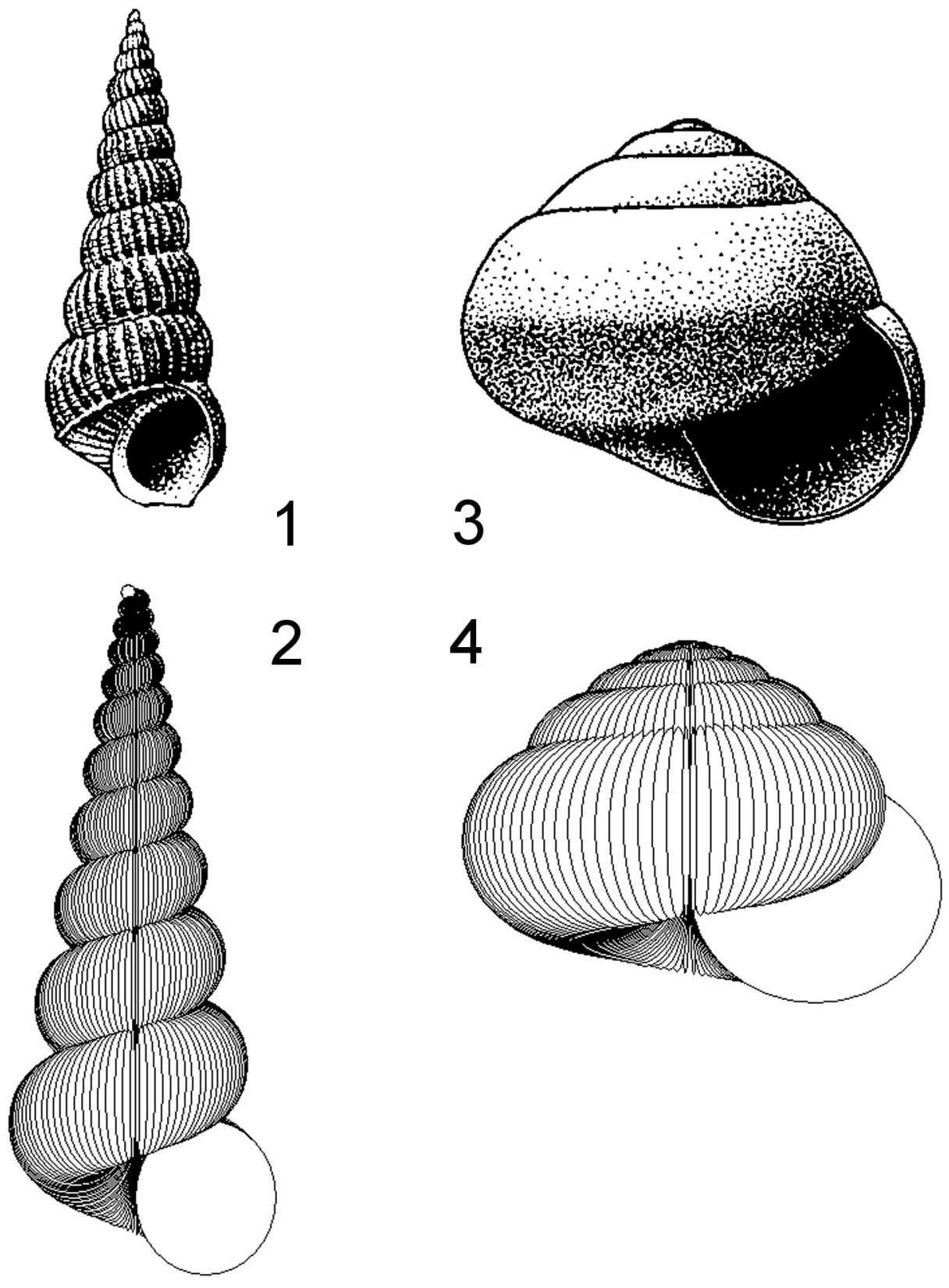

FIGURE 2. Isometric and allometric gastropods and simulations. 1, An example of a "Raupian" gastropod (Epitonium), showing isometric growth. 2, An example of a "Raupian" simulation with similar shape. 3, An example of an allometric gastropod (Megacochlea); note that there is no straight line that is tangential to the outside of all whorls. 4, A simulation using the program described here with similar shape. (Figures 2.1 and 2.3 (c) The Trustees of the Natural History Museum, London, reproduced from Anon, 1975, with kind permission.)

Progenesis. Reduce the number of revolutions about the coiling axis. The default reduction offered by the computer program is one revolution.

Neoteny. Slow the rate of ontogenetic shape change by reducing $\Delta W$ and $\Delta T$. The default change offered by the computer program is $x 3 / 4$.
Hypermorphosis. Increase the number of revolutions about the coiling axis. The default increase offered by the computer program is one revolution. Acceleration. Increase the rate of ontogenetic shape change by increasing $\Delta W$ and $\Delta T$ '. The 


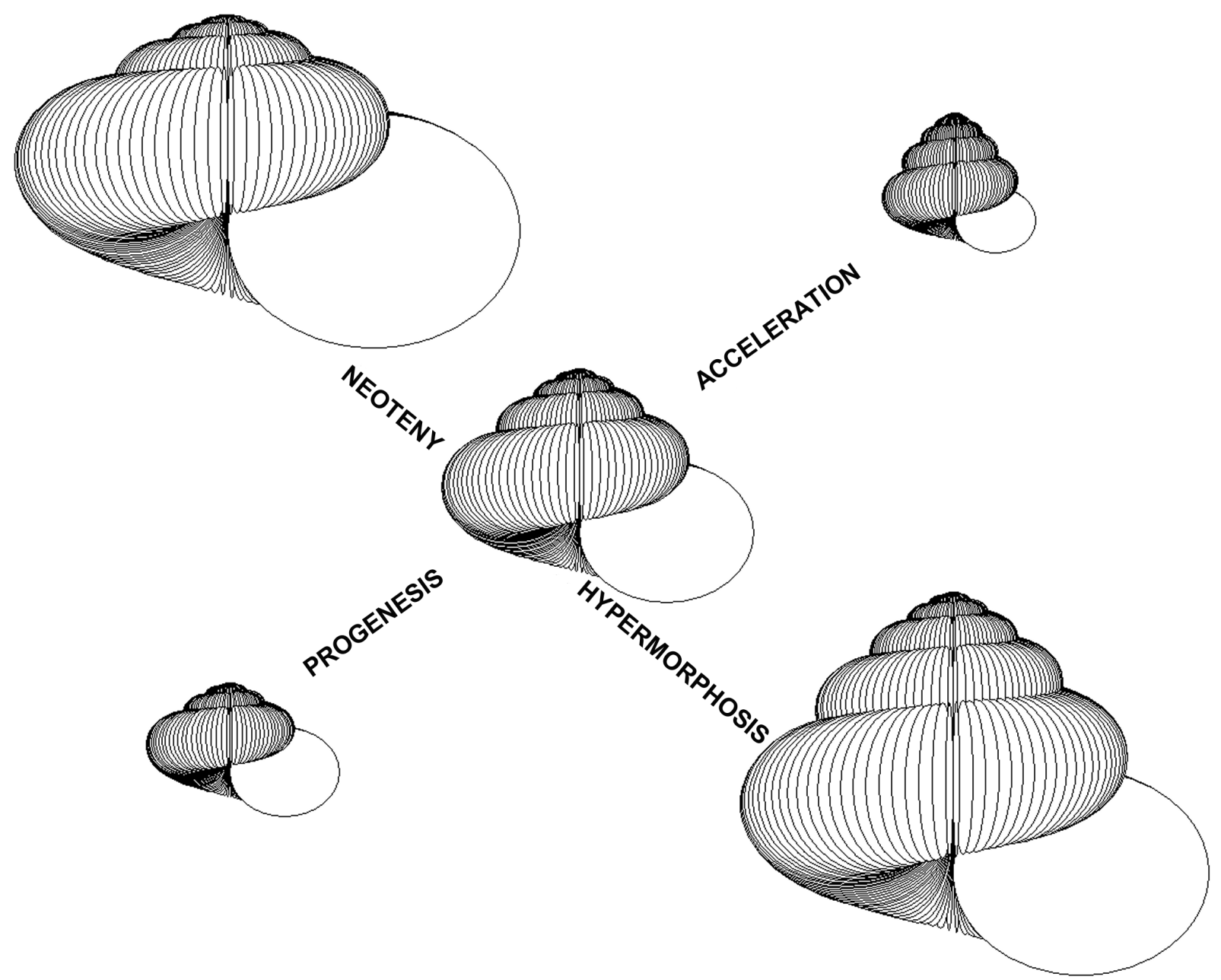

FIGURE 3. Modes of heterochronic change. The central simulation has parameters $W_{0}=2.5, \Delta W=-0.12, D=0, S=0.8$, $T_{0}^{\prime}=0.4, \Delta T^{\prime}=0.06$, and has six whorls. The other four simulations show the result of one increment of each mode of heterochronic change. The two forms on the left are paedomorphic; the forms on the right are peramorphic. Note that in this case neoteny results in larger size as the ontogenetic decline in whorl expansion is reduced, and acceleration results in a smaller size for the converse reason.

default change offered by the computer program is $x 4 / 3$.

Figure 3 shows the range of "descendants" that are simulated from an example allometric "ancestor" by one increment of each of the above modes.

The scope for experimentation with the model is infinite, and different parameters of the "ancestor" yield a variety of often surprising heterochronic "descendants". Figures 4, 5 and 6 show some illustrative examples.

\section{DISCUSSION}

\section{Hypotheses for Heterochronic Evolution}

The pattern of diversity among allometric gastropods, especially Pulmonata, is generally consistent with the range of forms that can be simulated by heterochronic change applied to typical shell shapes. For example, Figure 4 shows that the curious narrowing of the final whorls in fusiform and pupaeform gastropods can be predicted on the basis of evolutionary acceleration of negative ontogenetic change in whorl expansion and positive ontogenetic change in whorl translation (these ontogenetic trends being typical of "conservative" Pulmonata). However, this evolutionary change is certainly not thereby proved, because: 1) no 


$$
Q
$$



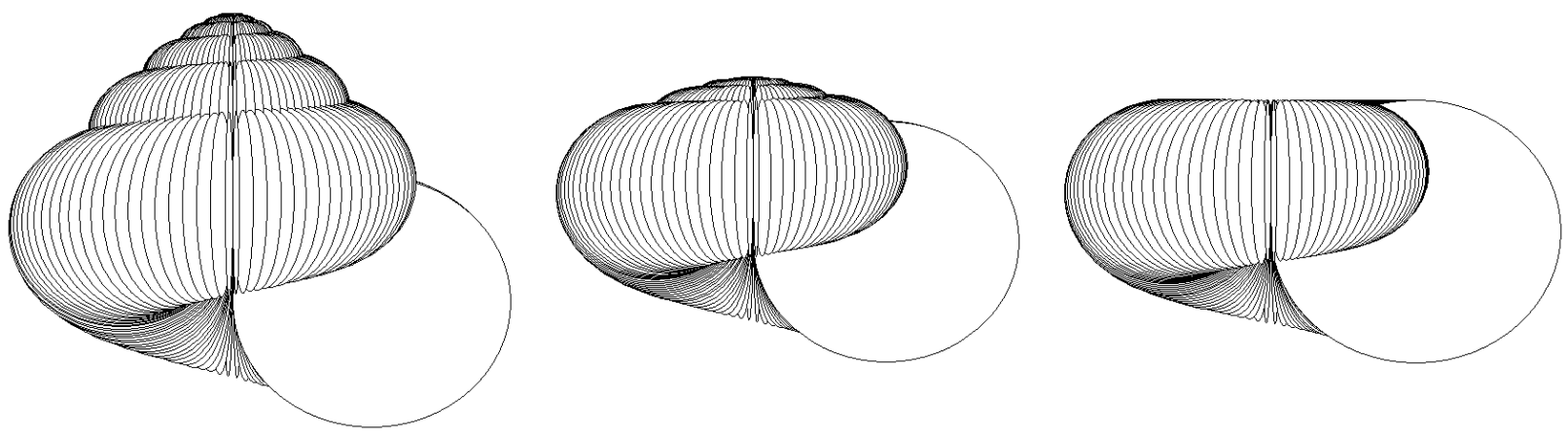

FIGURE 6. Heterochronic modelling experiment 3. The "ancestor" on the left has parameters $W_{0}=2, \Delta W=-0.05, D=0$, $S=0.9, T_{0}^{\prime}=0.2, \Delta T^{\prime}=0.07$, and has eight whorls. As in Figure 4, this is a common general type of gastropod shell shape. The central simulation is a "descendant" derived from this by two increments of neoteny (relative scale $x 0.5$ ); the simulation on the right has resulted from two further increments of neoteny (relative scale $x$ 0.39). All three simulations have analogues in the Family Helicidae of the Pulmonata, and this result constitutes a hypothesis for the possible heterochronic evolution of forms with flattened spires (depressed and sub-planispiral).

parison of carefully measured empirical ontogenies between known ancestors and descendants. Nevertheless, modelling experiments such as those described here are useful in developing ideas and formulating hypotheses that could then be tested with empirical data.

The demonstration of the wide range of forms that can be derived by increments of various heterochronic modes raises an important question: if real gastropods have evolved such shapes by heterochrony, are the morphologies an incidental outcome of selection for the heterochronic mode, or is heterochrony the means by which a species "finds" a favourable morphology? It is clear that alleles for earlier or delayed sexual maturity may be selected under certain circumstances, and, in allometric forms, these will produce progenetic or hypermorphic descendants, respectively. Gould (1977) argued for "ecological correlates" of heterochronic modes, and, for example, connected progenesis with exploitation of ephemeral resources. If this is the principal "driving force" of evolution for a particular species at a particular time, then the adult morphology of the resulting descendant will be determined only by the ontogeny of the ancestor, and not by the performance of that morphology in the environment. To take the case of Melo in Figure 5 , the shape might be an inadvertent consequence of selection for large size, effected via selection on alleles for faster rate of ontogenetic development. Alternatively, it could be that selection on the adult morphology is paramount, and this is attained, perhaps parsimoniously, by heterochronic exploitation of ontogenetic trends; hence, in Melo, acceleration might merely be the means by which a functionally favourable shape has evolved (with perhaps a low drag coefficient suitable for high-energy environments). It is certainly the case that ontogenetic allometry affords species the opportunity of the latter evolutionary route.

\section{Pedagogic Applications}

The demonstration of heterochrony is problematic in teaching palaeontology and evolution. Putative case studies are valuable but are not always convincing, because: 1) students need to observe and compare the ontogeny of ancestors and descendants, and clear illustrations are seldom available: and 2) theoretical models of what heterochrony would be predicted to produce are usually missing. Textbooks typically use illustrations of abstract morphologies in which arbitrary features join the ontogenetic sequence (the earliest being figure 7 in Gould, 1977). The current model can be used as a heuristic demonstration of the theoretical potential of heterochronic change, and students can compare simulations with real organisms as a first stage in assessing the validity of the heterochronic hypothesis. The author has used the model (in the form of the program HETEROSIM) in practical sessions in modules on palaeontology and evolution, and has supervised project work in which the program has been a key tool. Student feedback has been positive. Suggestions and advice on using the program are presented in Appendix 1, and a "screenshot" of the HETEROSIM program is presented in Appendix 2. 


\section{ACKNOWLEDGEMENTS}

The author is grateful for constructive criticism from the anonymous reviewers, which has resulted in significant improvements to this paper.

\section{REFERENCES}

Alberch, P., Gould, S.J., Oster, G.F., and Wake, D.B. 1979. Size and shape in ontogeny and phylogeny. Paleobiology, 5:296-317.

Allman, W.D. 1994. Patterns and processes of heterochrony in Lower Tertiary turritelline gastropods, U.S. Gulf and Atlantic coastal plains. Journal of Paleontology, 68:80-95.

Anon. 1975. British Caenozoic Fossils. Natural History Museum, London.

Gerber, S. 2011. Comparing the differential filling of morphospace and allometric space through time: the morphological and developmental dynamics of Early Jurassic ammonoids. Paleobiology, 37:369-382.

Gould, S.J. 1969. An evolutionary microcosm: Pleistocene and Recent history of the land snail Poecilozonites in Bermuda. Bulletin of the Museum of Comparative Zoology, 138:407-532.

Gould, S.J. 1977. Ontogeny and Phylogeny. Harvard University Press, Cambridge, Massachusetts.

Hammer, O. and Bucher, H. 2005. Models for the morphogenesis of the molluscan shell. Lethaia, 38:111122.

Kant, R. and Kullman, J. 1978. Gehause-Allometrie bei Cephalopoden. Neues Jahrbuch für Geologie und Paläontologie, 157:98-103.

McKinney M.L. (ed.) 1988. Heterochrony in Evolution: A Multidisciplinary Approach. Springer, New York.
Noshita, K., Asami, T., and Ubukata, T. 2012. Functional constraints on coiling geometry and aperture inclination in gastropods. Paleobiology, 38:322-334.

Okamoto, T. 1988a. Analysis of heteromorph ammonoids by differential geometry. Palaeontology, 31:35-52.

Okamoto, T. 1988b. Changes in life orientation during the ontogeny of some heteromorph ammonoids. Palaeontology, 31:281-294.

Raup, D.M. 1966. Geometric analysis of shell coiling: some general problems. Journal of Paleontology, 40:1178-1190.

Rice, S.H. 1998. The bio-geometry of mollusc shells. Paleobiology, 24:133-149.

Seuss, B., Nützel, A., Scholz, H., and Frýda, J. 2012. The Palaeozoic evolution of the gastropod larval shell: larval armour and tight coiling as a result of predation-driven heterochronic character displacement. Evolution and Development, 14:212-228.

Swan, A.R.H. 1988. Heterochronic trends in Namurian ammonoid evolution. Palaeontology, 31: 1033-1052.

Swan, A.R.H. 1999. Computer models of invertebrate morphology, p. 157-179. In Harper, D.A.T. (ed.), Numerical Palaeobiology. Wiley, Chichester.

Swan, A.R.H. 2001. The occupation of morphospace, p. 157-161. In Briggs, D.E.G. and Crowther, P.R. (eds.), Palaeobiology II. Blackwell Science, Oxford.

Tissot, B.N. 1988. Geographical variation and heterochrony in two species of cowries (Genus Cypraea). Evolution, 42:103-117.

Urdy, S. and Chirat, R. 2006. Snail shell coiling (re-)evolution and the evo-devo revolution. Journal of Zoological Systematics and Evolutionary Research, 44:1-7. 


\section{APPENDIX 1.}

Use of the program HETEROSIM, and exercises for students.

The computer program HETEROSIM constructs geometrical shapes using the equations presented in this paper. The graphical construction is achieved by drawing opaque ellipses to represent successive positions of the whorl, with those nearest the viewer drawn last to create a rough impression of a solid 3D form. This graphical style is regarded as being satisfactory, but close inspection does reveal minor anomalies in the vicinity of the coiling axis. Other authors have produced renderings that are smoother and with a technically superior 3D appearance (e.g., Hammer and Bucher 2005; Noshita 2012), but this is thought to be unnecessary for current purposes, and in some respects the graphical style used here is clearer. HETEROSIM is restricted to simulations with elliptical whorl sections; this is thought to be appropriate, as attempting to find fits to other whorl shapes would be time-consuming and not relevant to the task of modelling heterochronic change in gross shell shape.

The user of the program is presented with eight boxes containing values that can be userspecified prior to heterochronic modelling: these are the six parameter values described in this paper, plus the number of whorls and a relative scale factor (see Appendix 2). The default values are those used in Figure 4. Clicking on the "MODEL" button then generates the simulation using the current parameters. (N.B. The parameters defined in this paper as $W_{0}$ and $T_{0}^{\prime}$ appear on-screen as "W" and "T".).

The effect of heterochronic modes can then be simulated by clicking on the box(es) corresponding to the desired mode(s) (this will produce a "tick" in the box), and clicking on the "MODEL" button again. Repeated increments of the same or other modes can be executed. The modes can be de-selected by clicking on the ticked boxes. (For the sake of simplicity, and ease of use in a pedagogic environment, this version of the program offers only the default values for heterochronic change that are detailed in the main text of this paper.)

When the effect of heterochronic modes are modelled, the values of the parameters are updated. In one modelling experiment, it is expected that the user will not manually adjust the parameter values (except perhaps the scale factor) after setting-up the initial values.
The appropriate scale factor for the initial simulation is estimated by the program on the basis of the initial parameter values, but may nevertheless need to be adjusted. After applying heterochronic changes, the size of the simulated shell very often changes substantially. The scale factor can be adjusted readily by clicking on the " $\wedge$ " or " $v$ " buttons. When this is done, all current heterochronic mode selections are switched off.

There is no special provision for capturing and printing graphics; the familiar $<$ CTRL $><$ ALT $><$ PRNT SCRN $>$ key combination can be used, or import facilities in graphics editing software.

The program should work on all PCs with Microsoft Windows or Windows emulation. It may be found that the program doesn't initially run, and an error is shown that reports a missing Dynamic Link Library (.DLL) file. If so, this file can readily be found on the Microsoft web site and installed easily and free of charge. Users in European countries may need to change the decimal separator from a dot to a comma.

\section{Exercises for Students}

Initially, students should become familiar with the geometric parameters in the isometric case. Parameters $\Delta W$ and $\Delta T^{\prime}$ should both be set to zero, and $W, T, S$ and $D$ adjusted (one at a time) so that the effect of each can be noted (this is good practice in gaining familiarity with any computer simulation). This can lead to more structured investigation:

1. Students should be introduced to the concept of morphospace, and a two-dimensional array of forms could be produced in the style of figure 2.2.3.1 in Swan (2001), perhaps with $D$ and $S$ kept constant and simulations with various permutations of $W$ and $T$ ' produced.

2. Students can then be given the task of finding parameter combinations that yield simulations similar to actual specimens. Illustrations of a range of specimens could be presented; these could include one or two allometric forms so that the key difference can be observed. Students should find that the adjustments required in order to more closely simulate isometric forms is quite intuitive. The failure to simulate allometric forms is instructive.

3. Students could be invited briefly to experiment 
with heterochronic changes on isometric forms. They will observe that there is no change in shape, even when there is change of size (in the case of progenesis and hypermorphosis).

Students can then be given the task of optimising all six parameter values for a given real allometric form. Members of the Pulmonata are good for this, especially the more typical members of the Helicidae. This optimisation is likely to be difficult, as this constitutes a search of six-dimensional morphospace, without an easy intuition of the direction in which the optimum lies. This is typical of the difficulty encountered when undertaking optimisation using models with large-dimensional parameter space in general.

4. Using a set of parameter values supplied by the instructor (perhaps the ones used in Figures 4, 5 and 6 ), students can experiment with the effect of the heterochronic modes. (In order to return to the starting point, they should note that an increment of neoteny can be reversed by an increment of acceleration and vice versa, and likewise for progenesis and hypermorphosis). They should attempt to understand how the "descendant" forms have resulted from the modification of the "ances- tral" ontogeny.

5. For a more extended project, students could locate a source of illustrations of allometric gastropods from a specific taxonomic group (perhaps at Family level) and find the six optimal parameter values for one of the species (it is probably best to use one of the more conservative forms). A range of heterochronic "descendants" can then be produced for comparison with the variety of forms that actually occur. The plausibility of the heterochronic hypothesis for that group can then be critically assessed. (Note that, ideally, the phylogeny of the specimens in the chosen taxonomic group would be known, but this information is seldom available).

6. For more advanced study, students could be invited to consider the possible functional performance of the various forms and the ecology of the specified taxonomic group, and reach a verdict on whether heterochrony might be the means of producing a better-performing adult shape, or whether the "descendant" adult shape is merely a consequence of the favourability of evolutionary change in rates and timings in ontogenetic development. 


\section{APPENDIX 2.}

"Screenshot" of the HETEROSIM program, as presented to the user.

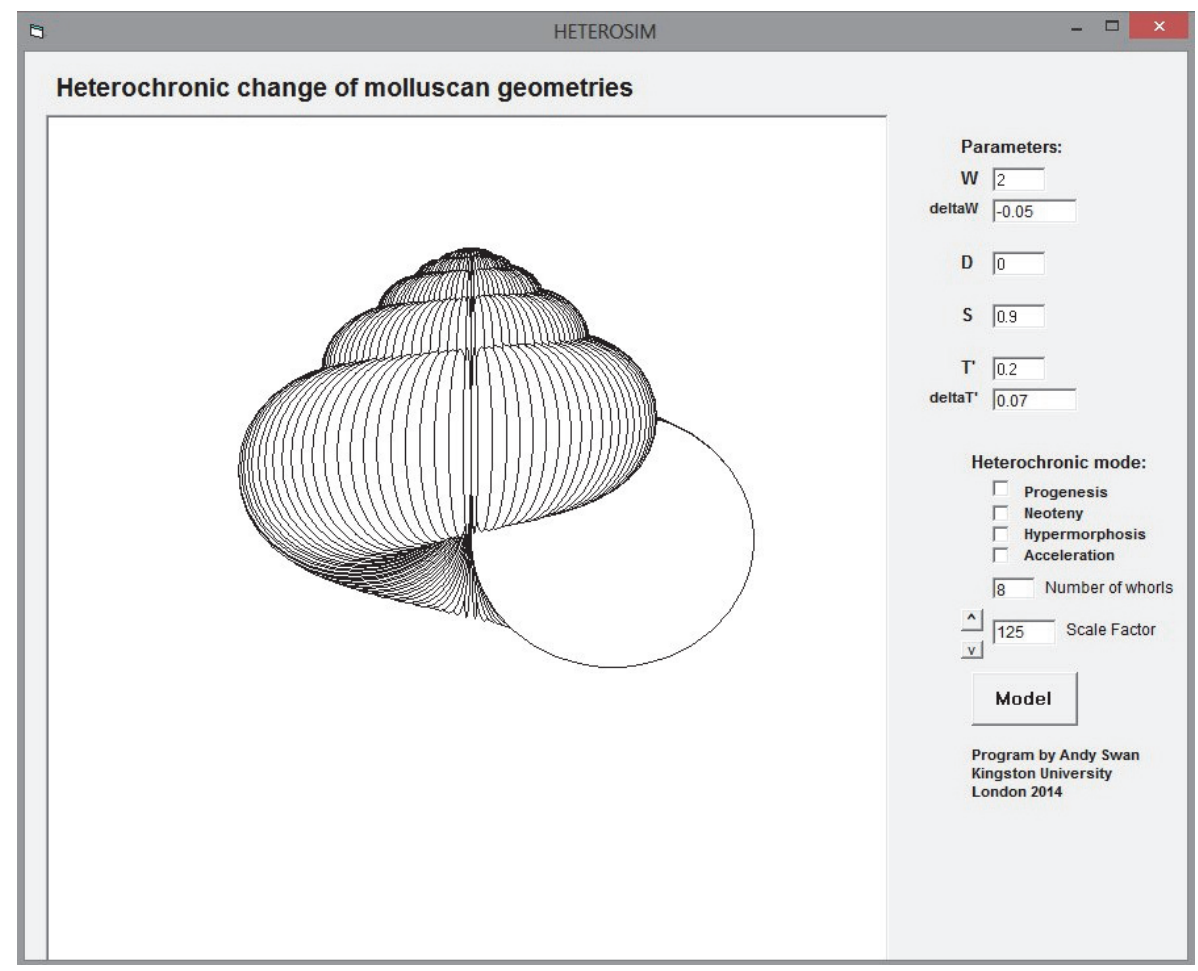

